\title{
PERSPECTIVE RESTITUTION FROM VIEW CAMERAS PHOTOS
}

\author{
Fabrizio Agnello
}

University of Palermo, Dept. of Architecture, fabrizio.agnello@unipa.it

Commission II

KEY WORDS: Perspective restitution, Single image photogrammetry, Period photos, View Camera, Virtual reconstruction.

\section{ABSTRACT:}

The paper aims at discussing the accuracy of perspective restitution from view camera photos; view cameras are non-standard cameras frequently used in the past century for on field shooting of buildings and urban sites; this is why the reconstruction of lost buildings often deals with photos taken with a view camera. The case study chosen for the proposed experiment is an urban complex built in Palermo in the '50s. The site features a very regular layout with surfaces at right angle, that supports the graphic reconstruction of photos' inner and outer orientation. The site has been surveyed with a laser scanner; the point cloud provided the metric information needed for the evaluation of the accuracy of perspective restitution. The experiment used three sets of photos: a photo taken by Studio Alinari in the '60s; a couple of photos taken during the research work with a view camera; a photo taken with a standard mirrorless camera.

The results of the experiment prove that view cameras do not modify the projective layout of perspective and that the accuracy of restitution from view camera photos is comparable to the accuracy of restitution from images taken with a standard camera.

\section{INTRODUCTION}

Digital surveying and representation make perspective restitution from photos an effective tool for the reconstruction of buildings that no longer exist and are pictured in a single or in few period photos (Fig. 1).

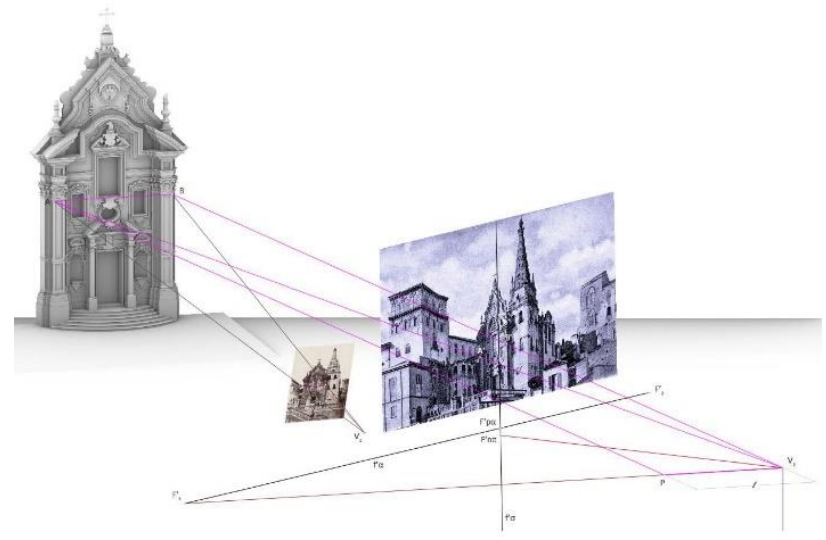

Fig. 1: 3D perspective restitution from period photos.

Perspective restitution is the simplest and less accurate photogrammetric technique; it is rooted in descriptive geometry as the inverse problem of perspective.

Perspective restitution is a neglected technique: it is not even mentioned in manuals of photogrammetry, surely due to its lack of accuracy; it is ignored or briefly discussed in manuals on descriptive geometry as well, since it is nothing more than the inverse problem of perspective.

In the pre-digital era perspective restitution has been applied to the restitution of the projective layout of perspective drawings, especially of trompe l'oeils and anamorphic paintings. It has rarely been used for the restitution of buildings from photographic images, because such restitution resulted dramatically inaccurate.

Perspective restitution from photos is actually almost different from perspective restitution of drawings. In perspective drawings, vertical edges are usually represented by vertical parallel lines, since architects and painters preferred using a vertical picture plane, that makes perspective construction easier. In photos, the picture plane is the camera's sensitive flat surface (sensor, film or plate) that captures the image; in conventional cameras the shooting axe, i.e. the axis of the lens, is perpendicular to the sensitive plane. If the photographer aims at capturing the image of a building with the vertical lines parallel to each other, the camera has to be set on a levelled tripod and the shooting axe has to be perfectly horizontal; it is easy to suppose that this condition is almost impossible, especially when photos are taken on field.

This is why vertical lines are rarely parallel to each other in photographic images and meet in a vanishing point. Furthermore, the lines that are at right angle with the picture plane are not horizontal and their vanishing point, i.e. the perspective principal point, will not lie on the horizon line.

Even when photographers aim at mitigating the convergence of vertical lines, they do not fully succeed in this purpose; this is why the vanishing point of vertical lines is often at a remarkable distance from the image frame.

Digital representation tools overcome many of the limitations that made perspective restitution inadequate to the reconstruction of buildings depicted in photographic images; it is worth mentioning that one of the limitations of pre-digital restitution precisely regarded the identification of the vanishing point of vertical lines.

The virtually infinite dimension of the digital drawing desk allows detecting even the furthest vanishing point and thus supports the graphic reconstruction of the inner orientation of the image.

In conventional photographic images the principal point can be easily retrieved at the intersection of the diagonals of the rectangular frame.

This location results sometimes in contrast with the perspective graphic reconstruction of inner orientation and the principal point results located in unexpected positions.

When the photo is taken from a book or a review, the eccentricity of the principal point is usually due to trims that are often made to make an image fit the editing layout.

In some experiments where the restitution process was developed on full format, untrimmed photos, taken in the first decades of the past century, the inner orientation process produced the principal point in an eccentric position. 
This result suggested further investigations.

\section{RELATED WORKS}

The interest in perspective restitution from photos aroused, after a long silence, at the end of the ' $90 \mathrm{~s}$, along with the initial dissemination of PC. Scholars in computer engineering tried to develop automated processes for line extraction, vanishing point identification, intrinsic and absolute orientation (Debevec, 1996, Van den Heuel, 1998). In recent years further studies aimed at the automated extraction of 3D textured models from an image; software tools were announced and discussed in publications (Arslan, 2014), but no evidence of their use in further researches has resulted.

Scholars in descriptive geometry use perspective tools to calculate the intrinsic and absolute orientation of photos (Fallavolita et al., 2013, Dzwierzynska, 2017), but no use of 3D modelling tools is proposed or discussed (Ramon-Constanti and Gomez, 2020).

No specific studies on perspective restitution from view camera photos have been retrieved by the author.

\section{VIEW CAMERAS}

A view camera is a peculiar type of camera that was largely used in the first decades of the photographic era and is still used today by professional photographers and photography scholars.

Many photos that picture buildings that no longer exist, due to war events, natural disasters or urban renewal programs, were taken with a view camera.
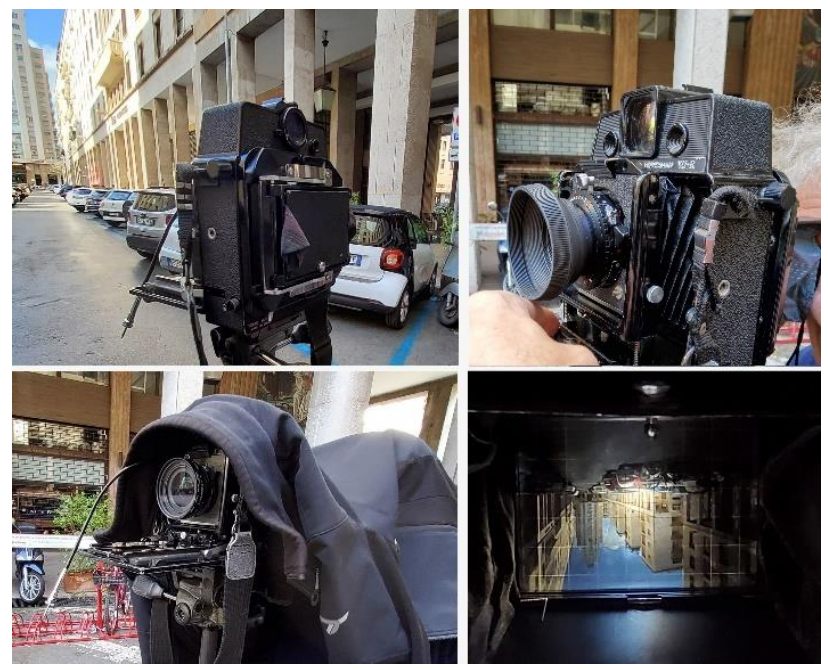

Figure 2: The view camera used for the experiment.

A view camera allows, before capturing the image, a precise, real time control of focus, depth of field, framing and perspective; digital photography can operate post process perspective corrections, but in the pre-digital era, save minor adjustments during the printing process, the quality of an image was mainly due to the control in the acquisition step.

A view camera (Fig. 2) echoes the prototypes of the beginnings of photography, where a dark room, made by a box, was pierced by a hole that allowed the light inside and hosted the surface that received the light and produced the image.

In the view camera the dark room makes the connection between the lens (the evolution of the hole) and the sensitive surface (film, sensor or plate); in order to allow the movements of the lens and the sensitive surface, the dark room is made by a flexible bellow. The element that holds the sensitive surface, i.e. the film, sensor or plate, is named 'rear' standard, whereas the lens holder is the 'front' standard; the standards are connected to a rail that allows to adjust their distance.

Depending on the view camera model, lens and film holders can make the following movements: i) up and down, right and left translations; ii) swing, i.e. rotation around a vertical axe; iii) tilt, i.e. rotation around a horizontal axe (Fig. 3).
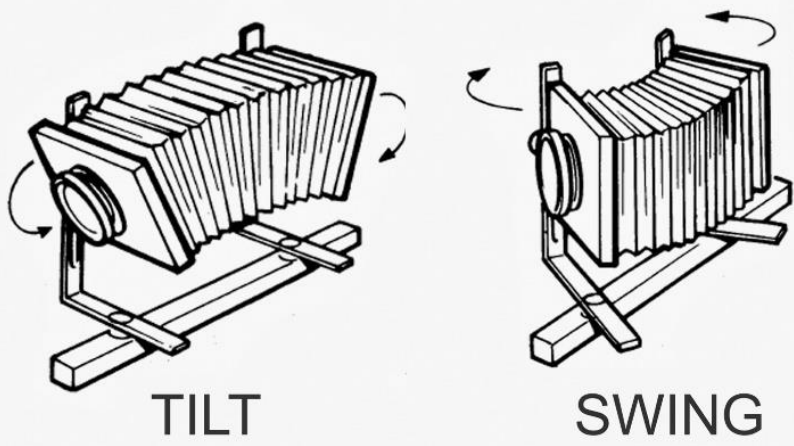

Figure 3: Rotation of front and rear standards around a horizontal axe (tilt) and a vertical axe (swing).

The translation of front and rear standards modifies the framing and allows to shoot parts of the real scene that would be trimmed out by a conventional camera.

The rotations of the front standard, the lens holder, adjust the focus and the depth of field.

The rotations of the rear standard, the film holder, modify the perspective, since they change the orientation of the picture plane.

The rotation around the vertical axe (swing) changes the perspective of horizontal lines, whereas the rotation around the horizontal axe (tilt) changes the perspective of vertical lines. Photographers that shoot buildings and urban sites with a view camera often use the rear standard's tilt to mitigate or remove the convergence of vertical lines, especially when the subject is a very high building, or a building that is placed on an elevated spot. Today view cameras are mainly used in photographic studios, but in the first half of the past century they were often used to shoot monuments and monumental sites.

Two photos of the church of San Gregorio in Messina (Sicily), taken few years before the devastating earthquake that destroyed the town in 1908, clearly illustrate the effect produced by rear standard's tilting (Fig. 4).
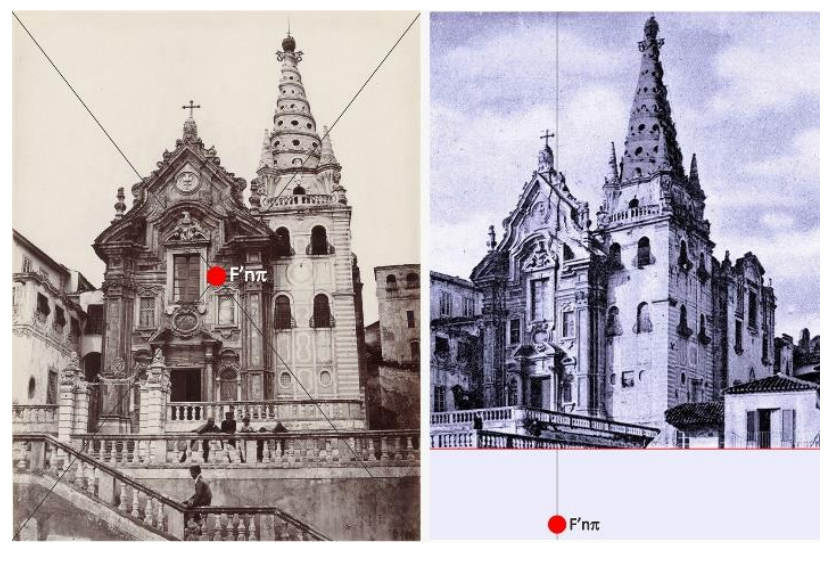

Figure 4: Photos of the lost church of San Gregorio in Messina, taken with a standard camera, or a view camera in neutral position (left) and with a view camera having the rear standard tilted (right). 
The church is located on a hill not far from the centre of the town and all the buildings and roads around the church are sited at a lower elevation.

In the first image (Fig. 4, left) the view camera is probably in a neutral position and the whole camera has been tilted to frame the entire façade; in this image vertical lines converge to a vanishing point, not far above the image frame and the principal point is located at the intersection of the diagonals of the image frame. In the second image (Fig. 4, right) the vertical lines appear parallel to each other, but they actually converge to a vanishing point placed far away below the image frame.

The vanishing point of vertical lines appears below the image frame when the shooting axe points downwards; it is reasonable to argue that in this circumstance the photographer presumably exaggerated the rear standard tilt correction, thus inverting the perspective layout.

The first image has been retrieved in its full format, whereas the second one has been taken from a book. In the second image the principal point is eccentric both to the vertical and to the horizontal symmetry axe of the image frame. No data allow to exclude that the eccentricity of the principal point is due to editorial trims, but the knowledge of the orography of the site, documented by period drawings, proves that a photo of the entire façade with a horizontal shooting axe was not possible at that time; this stands for the hypothesis that the reduction of the convergence of vertical lines is due to the rear standard's tilt.

The question that this paper aims at discussing is: do perspective corrections generated by the rotations of view cameras' rear standard modify the projective structure of perspective? Can images taken with a view camera be used for reconstruction purposes? The question is almost relevant, because many buildings and urban sites that no longer exist have been pictured with view cameras in the first half of the past century.

\section{PROPOSED METHODOLOGY}

In order to answer the proposed questions, a peculiar urban site has been chosen and has become the test bed for the experimentation (Fig. 5).

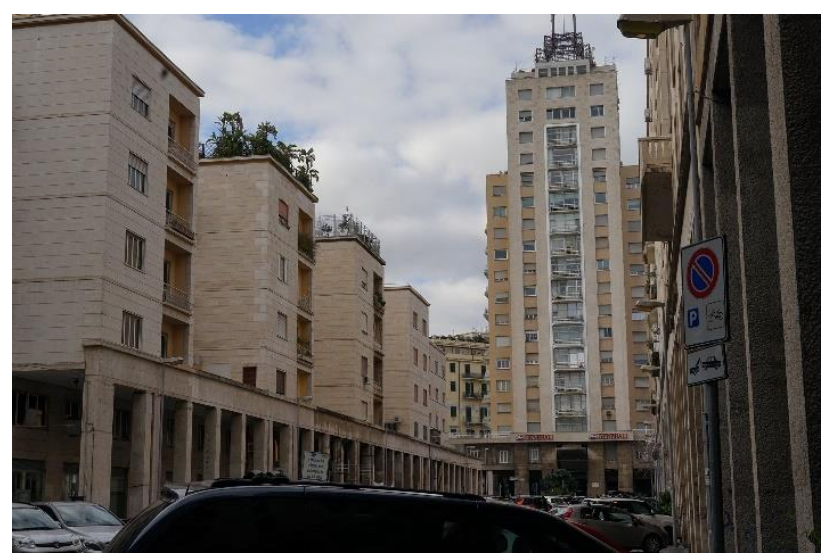

Figure 5: The inner courtyard of the urban complex.

The site is an urban complex designed and built in the ' $50 \mathrm{~s}$; the features that make this site a good test bed for an experiment on perspective restitution are: i) the wide open space at the centre of the site is encompassed by buildings that form a perfect perspective scene, with orthogonal lines and planes, and is terminated by a high regular building; ii) a photo of the site, commissioned to the photographic studio Alinari, was taken in 1962, few years after the end of the construction works; in this photo, taken from the ground as evidenced by the perspective, the vertical lines of the high building appear almost parallel and this leads to argue that the photo was taken with a view camera.

The first step of the experimentation addressed the laser scanning and SfM photogrammetric survey of the site. Six scans have been taken and the point cloud resulting from the registration process has been rotated to make the front of the tall building parallel to $\mathrm{xz}$ reference plane. Photos have been aligned and the photogrammetric model has been scaled and oriented with the aid of the coordinates of peculiar points extracted from the laser scanning point cloud.
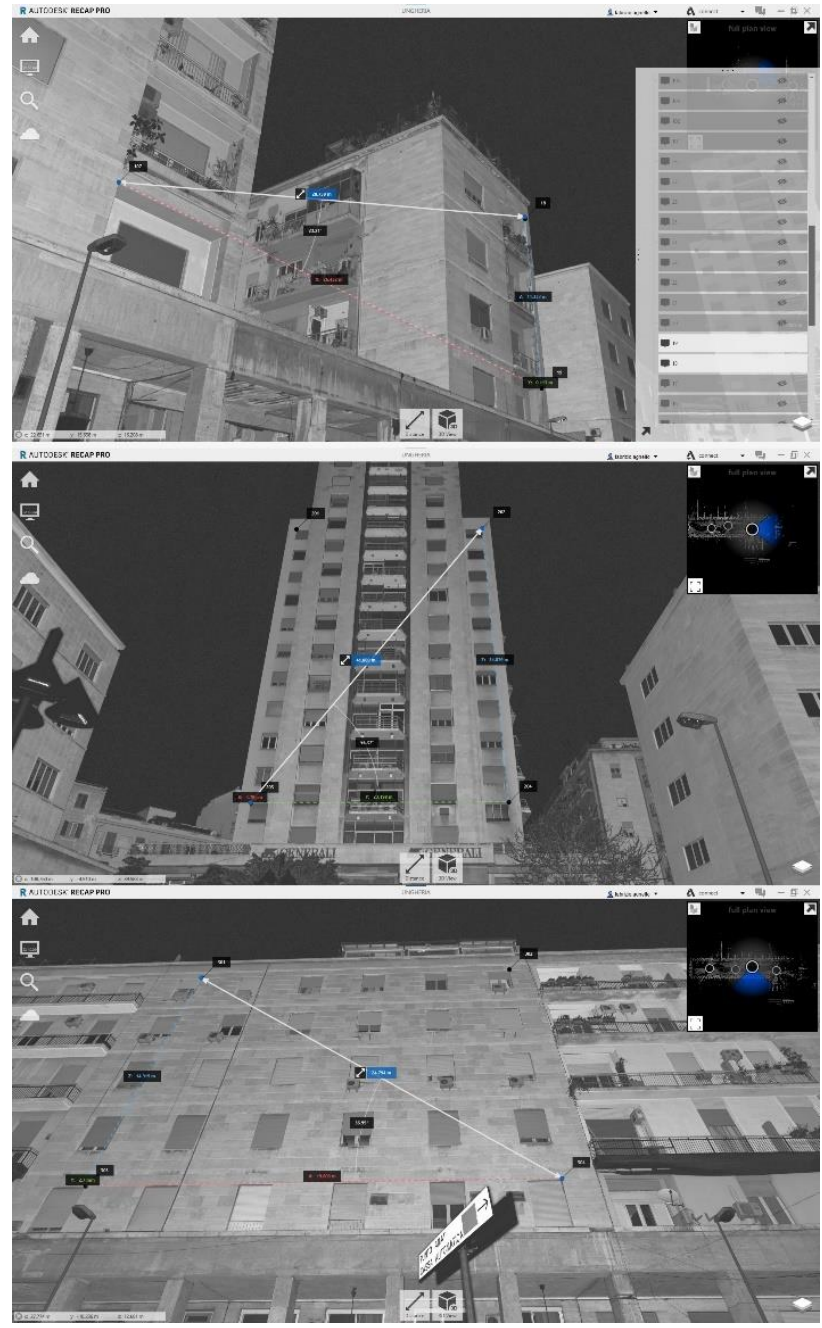

Figure 6: The rectangles used for the comparison with perspective restitution on the left front (above), on the high building (centre) and on the right front (below).

The point cloud provided the linear and angular dimensions that have been used for the restitution of the view camera photos' inner and outer orientation.

At a later stage, the point cloud tested the affordability of perspective restitution, applied both to conventional cameras and to view camera images.

For the purpose of the proposed experiment three photosets were used: i) the Alinari image; ii) two images taken for the experimentation with a view camera, courtesy of a photography scholar and teacher at the Academy of Arts of Palermo; iii) an image taken with a standard mirrorless camera.

The point cloud supported the extraction of the vertexes of windows or openings that detect the following shapes: two rectangles on the left longitudinal front; one rectangle on the front 
of the high building and one rectangle on the right longitudinal front (Fig. 6).
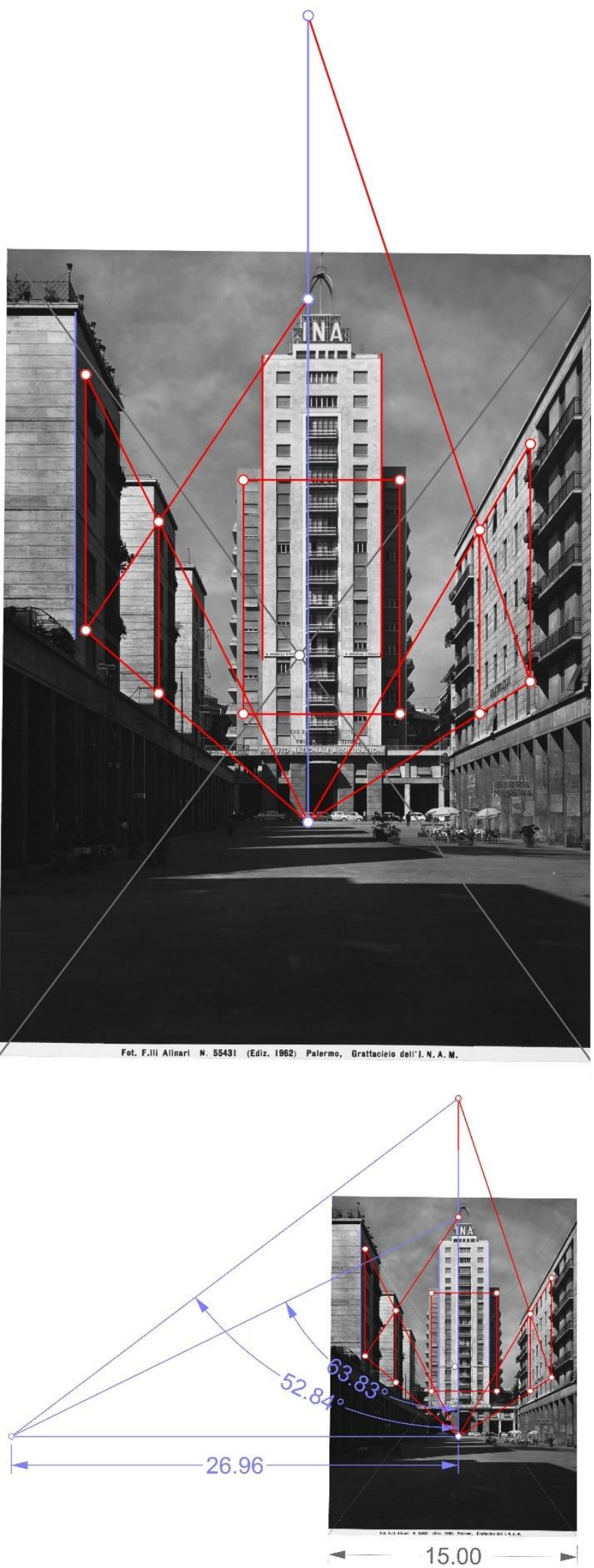

Figure 7: Photo Alinari with the rectangles highlighted (above); perspective reconstruction of the inner orientation (below).
The restitution of the inner orientation of the foto taken by Alinari followed a workflow that is almost unusual in perspective restitution from photos.

The photographer succeeded in correcting almost at perfection the convergence of vertical lines; hence, the vanishing point of vertical lines is at infinity and cannot support the reconstruction of the inner orientation.

At the same time, the image shows an almost perfect parallelism of the horizontal lines of the high building.

The lack of vanishing points is a real problem in perspective restitution but, in this case, the difficulty was overcome thanks to the available info referred to the image and to the depicted scene. The photo, purchased from the Alinari store, has preserved its full format (glass photographic plate $15^{*} 21 \mathrm{~cm}$ ); unluckily, no info about the focal length are available.

The image has been rotated to make the vertical lines parallel to $y$ axis and hence the horizontal lines of the high building parallel to $\mathrm{x}$ axis; in this peculiar condition the horizontal lines of left and right front result perpendicular to the picture plane (the photographic plate) and their vanishing point is actually the principal point of perspective. As expected, this point does not match the intersection of the diagonals of the image frame, but is placed downwards (Fig. 7).
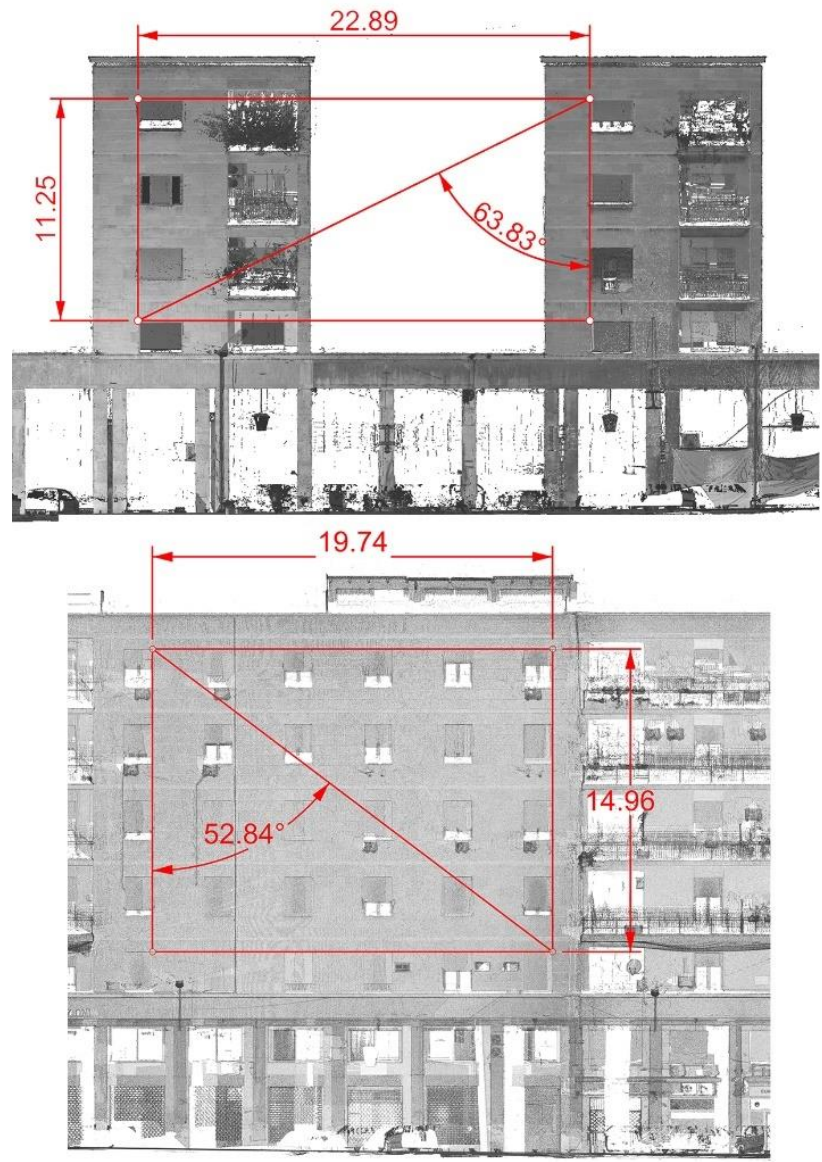

Figure 8: Rectangles and angles from the left (above) and right front (below), extracted from the laser scanning point cloud.

In order to reconstruct the principal distance, the diagonals of the rectangles of the left and right fronts of the open space were extracted (Fig. 8); the angles formed by these lines and the vertical sides of the rectangles allowed the reconstruction of the principal distance (Fig. 7); one angle would have been enough for such calculation, but the two allowed the evaluation of the 
error; in this case the error resulted almost irrelevant; using one angle for the calculation, the second one resulted 0.004 degrees different than the real one. The principal distance, i.e. the focal length, resulted equal to $269.6 \mathrm{~mm}$, a measure that is compatible with $270 \mathrm{~mm}$ lenses used by view cameras.

Once the inner orientation has been calculated, the restitution process was developed directly in 3D space, following a workflow discussed in previous papers. All rectangles have been restituted from the image and were compared to the homologous rectangles extracted from the laser scanning point cloud.

The results are really impressive. The difference between the real and the restituted shapes ranges from 5 to $30 \mathrm{~cm}$, an optimal result for perspective restitution, especially if compared to the size of the rectangles, whose sides range from 11 to $22 \mathrm{~m}$ on the sides and to $35 \mathrm{~m}$ in the high building. The perspective model has then been moved to superimpose the restituted left front rectangle to the real one; the verification of the accuracy of the restitution has been led through the measure of the distance between the calculated and real position of the two rectangles on the high building and on the right front: the restituted rectangle of the high building resulted $27 \mathrm{~cm}$ forward the real rectangle, whereas the rectangle on the left front resulted $19 \mathrm{~cm}$ far forward the real one; this error can be considered more than acceptable for the purposes of perspective restitution (reconstruction, visualization of monumental sites), especially if compared to the distance of the high building from the shooting point, almost 150 meters (Fig. 9).

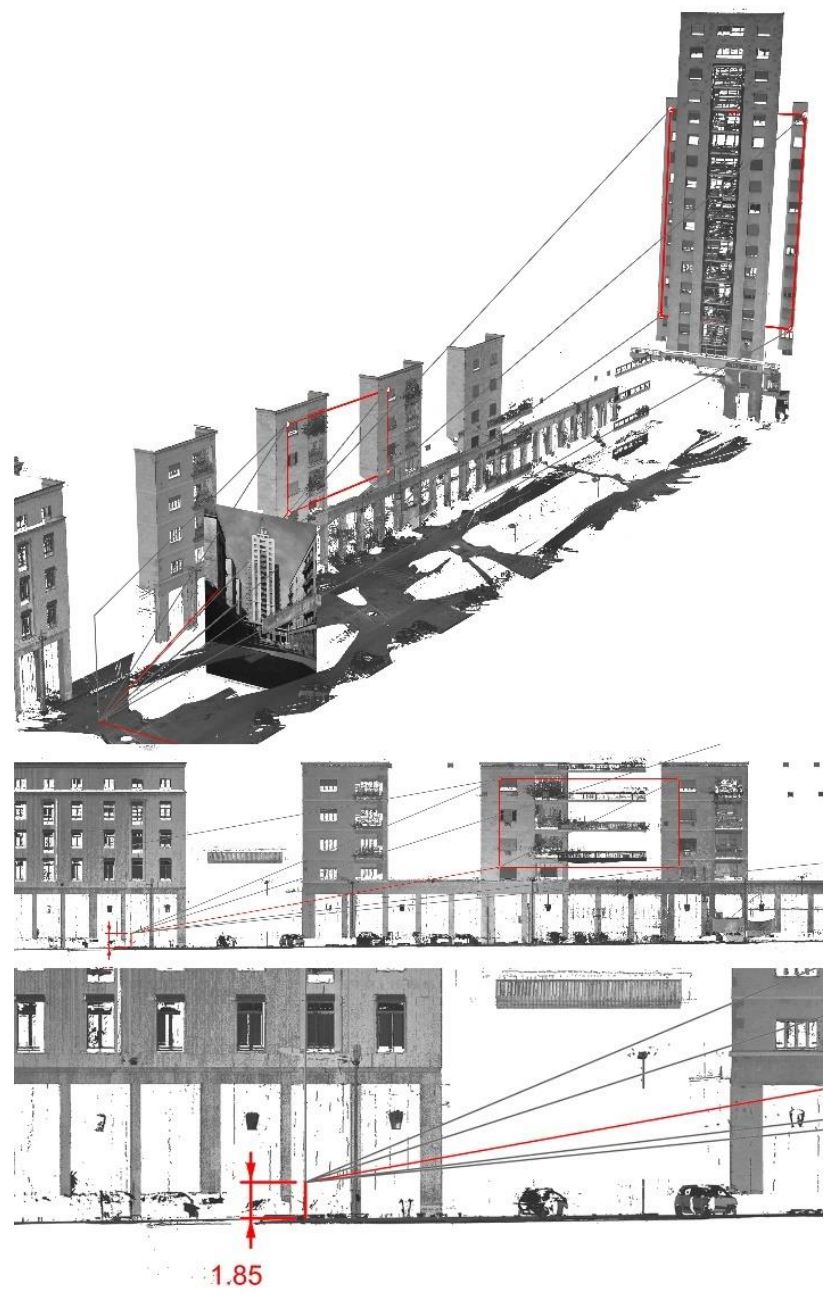

Figure 9: External orientation of Alinari's image and comparison between restituted and real rectangles; the position of the point of view results acceptable.
One of the relevant features of digital 3D perspective restitution is actually the capability in calculating the position of the point of view inside the real scene.

Such feature is very important, because it provides a further verification of the accuracy of the restitution process. If, for instance, the calculated point of view appears below the ground or in any other position not compatible with the morphology of the site, then the perspective restitution model should be reconsidered.

The point of view of Alinari's image appeared at the end of the open space opposite the high building, at a distance from the ground equal to $1.85 \mathrm{~m}$, surely higher than the real one, but not so far (Fig. 9).

The following step addressed the restitution of a couple of photos $(9 * 6 \mathrm{~cm}$ film, landscape orientation), taken for the experiment with a view camera mounting a $58 \mathrm{~mm}$ lens.

The first image has been taken in a neutral position, i.e. with no movements of the front and rear standards. In order to frame the high building, the whole camera has been tilted upwards and the image shows an evident convergence of vertical line (Fig. 10).

The second image was taken from the same point of view, with the whole camera in the same position; the rear standard has been tilted to mitigate the convergence of vertical lines and the image has been refocused. The correction was presumably exaggerated and the vanishing point of vertical lines appeared at a great distance below the image (Fig. 10).
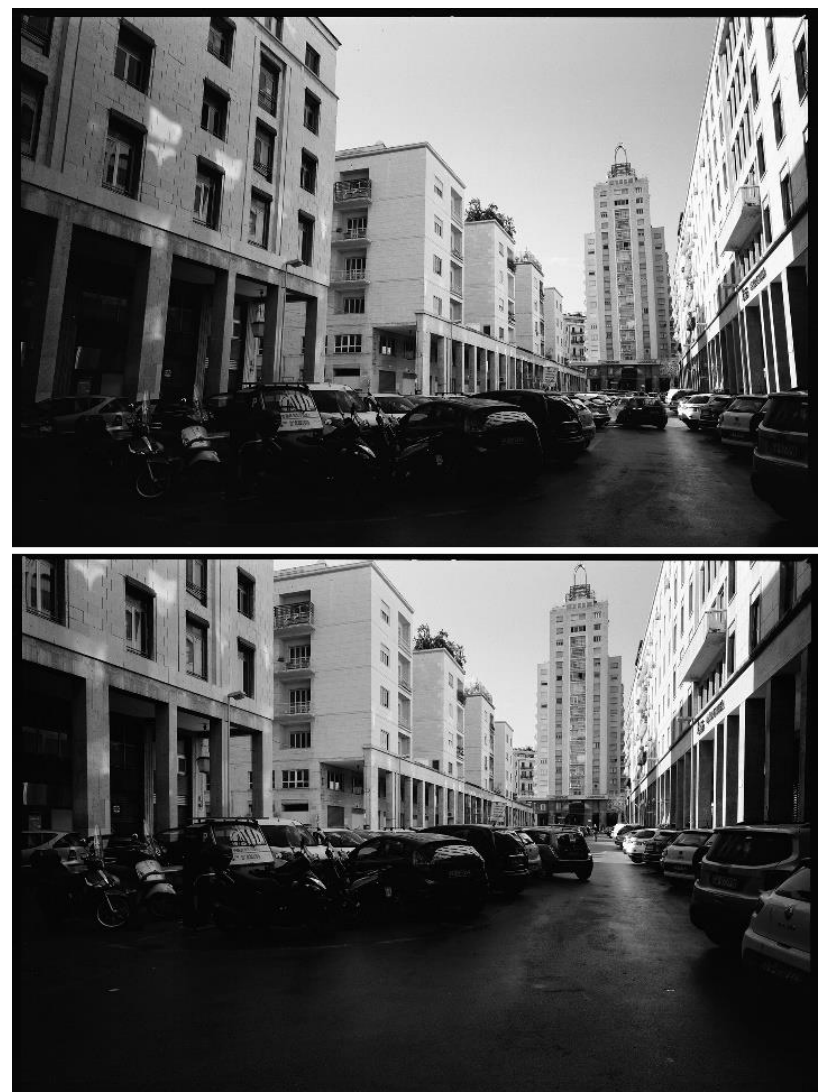

Figure 10: Photos taken with a view camera in neutral position (above) and with the rear standard tilt correction (below).

The calculation of the inner orientation evidenced a good approximation of the focal length, that resulted equal to $62 \mathrm{~mm}$ in the first image and $61 \mathrm{~mm}$ in the second one.

Once accepted the results of the inner orientation calculation, the restitution process followed the steps reported above. 
The position of the principal point did not match the expectations, since the distance of this point from the barycenter of the image resulted not so different in the two images.

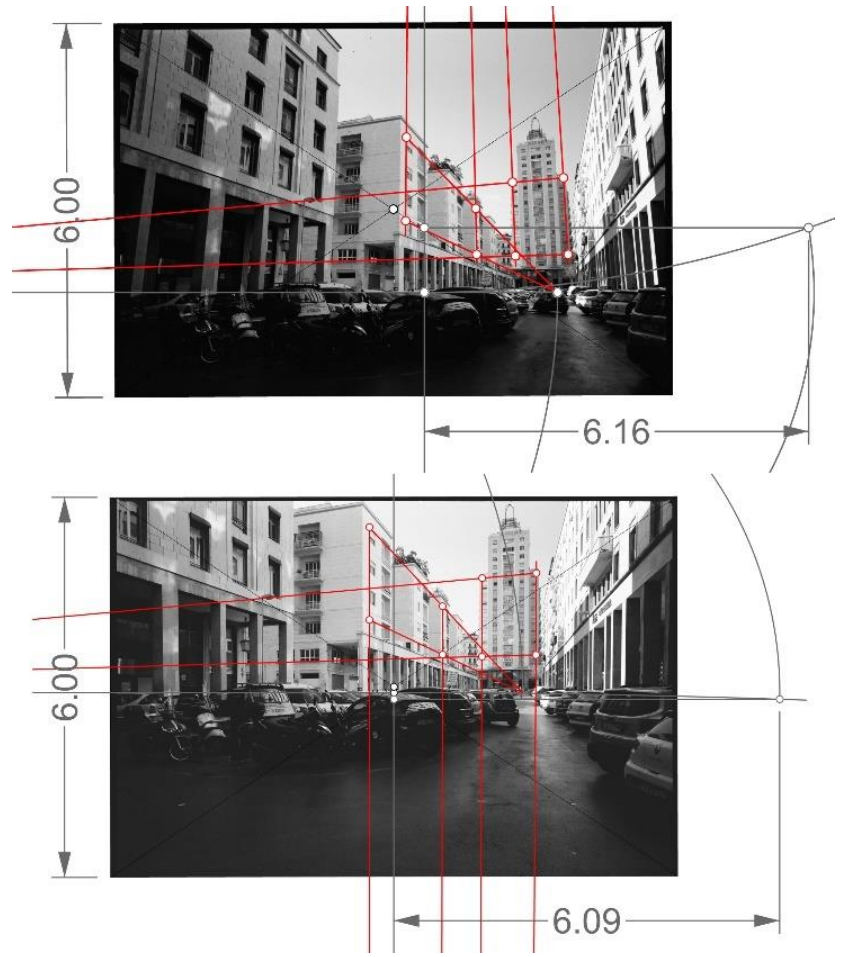

Figure 11: Inner orientation of View Camera's images.

This occurrence will probably be the subject of further future investigations (Fig. 11).

In these images the right front is not framed, so the restitution was restricted to the rectangle of the left front and to the rectangle of the high building.

The error in the restitution of the size of rectangles from the first image ranges from $10 \mathrm{~cm}$ in the left front to $43 \mathrm{~cm}$ in the high building. The position of the rectangle of the high building, resulting after the superimposition of the rectangles of the left front, resulted $45 \mathrm{~cm}$ backwards; it is useful to recall that the distance between the camera and the building is almost 180 meters (Fig. 12).
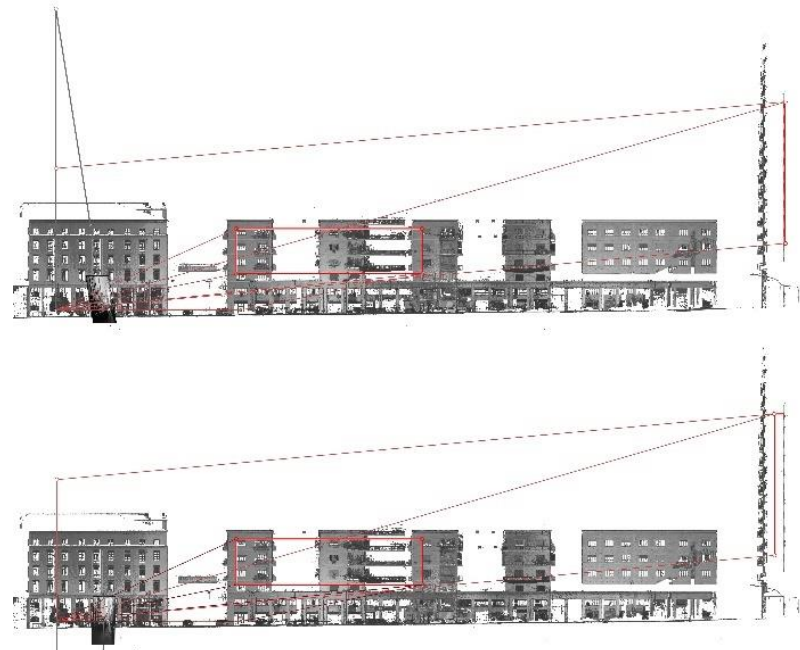

Figure 12: Front view of the external orientation of View Camera's images in neutral position (above) and with the rear standard tilt correction (below).
In the second image the errors range from $6 \mathrm{~cm}$ in the left front rectangle to $49 \mathrm{~cm}$ in the high building rectangle; the position of the restituted rectangle of the high building results $2.25 \mathrm{~m}$ forward the real one (Fig. 13)
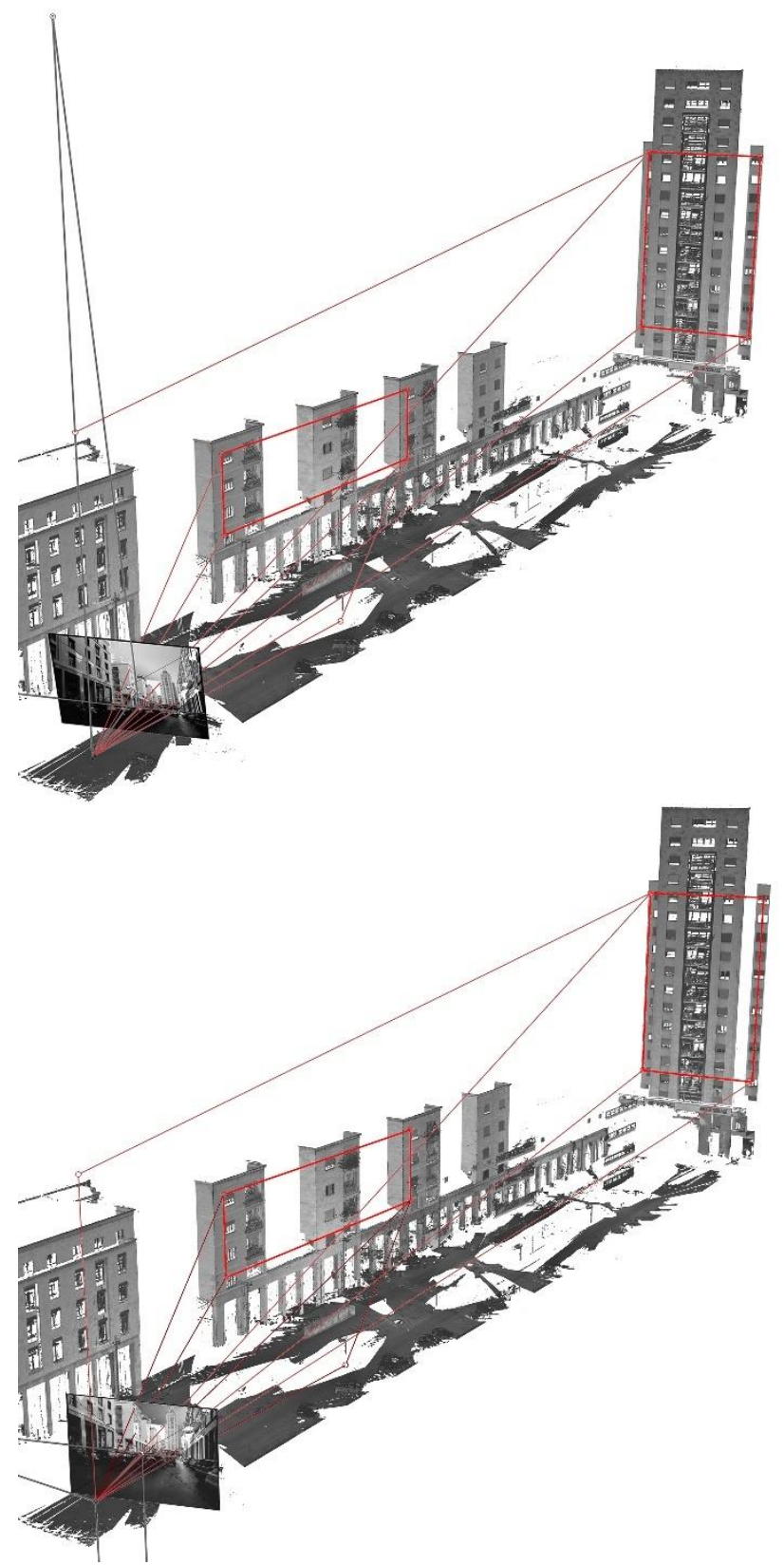

Figure 13: External orientation of View Camera's images in neutral position (above) and with the rear standard tilt correction (below).

The location of the points of view matches the real position of the camera in top view. The points of view of both images should perfectly match, since the images have been taken from the same spot; the distance from the restituted points of view resulted: $\mathrm{dx}=22 \mathrm{~cm}, \mathrm{dy}=19 \mathrm{~cm}, \mathrm{dz}=6 \mathrm{~cm}$.

The height of the points of view from the ground resulted respectively 1.63 and $1.69 \mathrm{~m}$, at all compatible with the real position.

The final test was developed with a standard image, captured with a mirrorless camera equipped with a $35 \mathrm{~mm}$ lens (Fig. 14). 


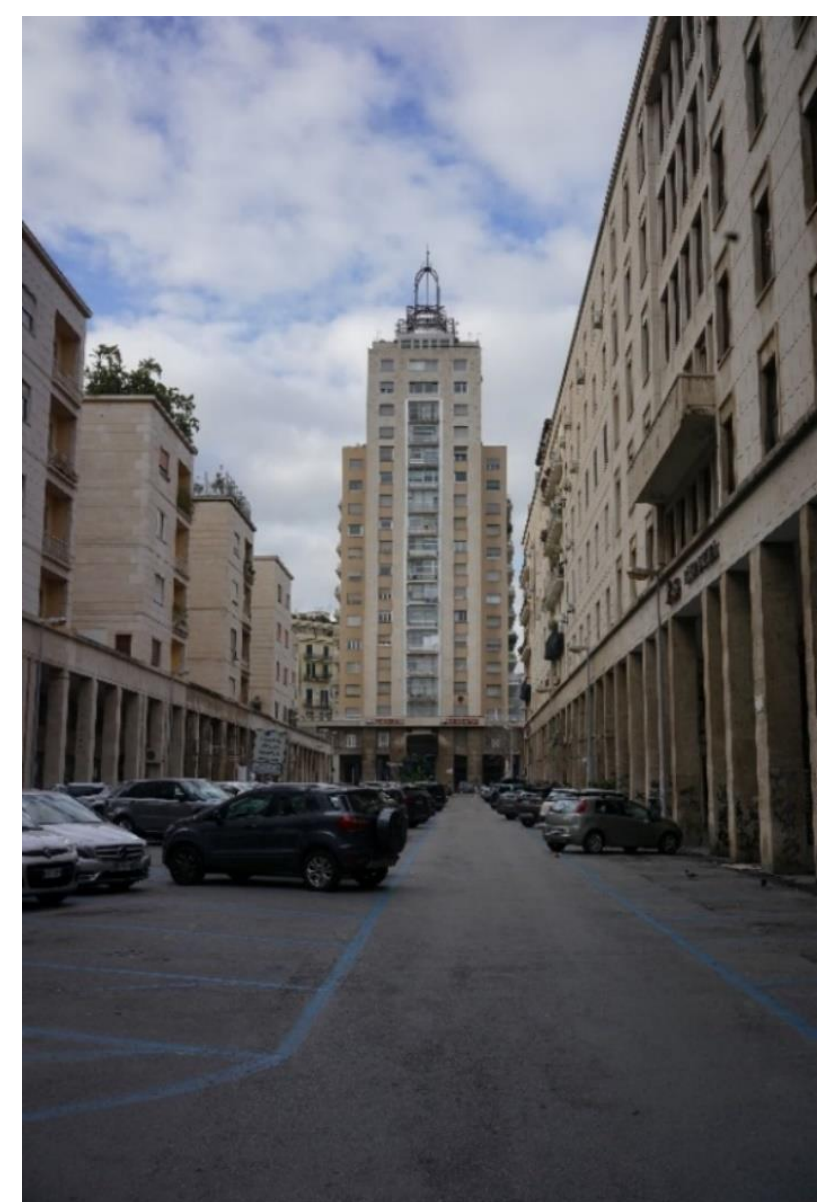

Figure 14: Image taken with a standard mirrorless camera mounting a $35 \mathrm{~mm}$ lens.

The calculation of inner orientation resulted $35.1 \mathrm{~mm}$ and the principal point matched the barycenter of the image (Fig. 15).

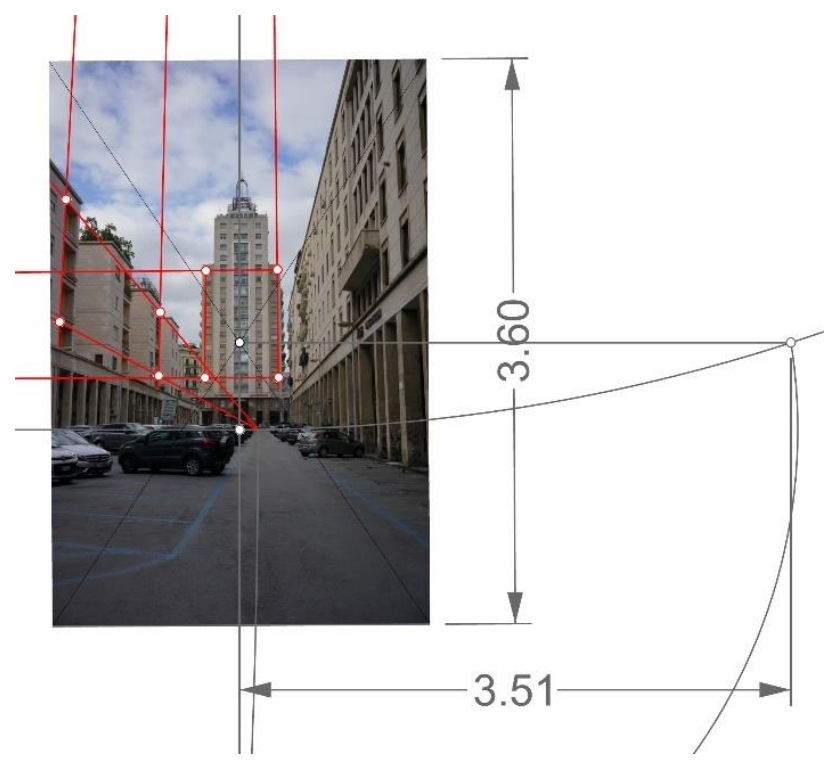

Figure 15: Inner orientation of the image taken with a standard mirrorless camera.

The height of the restituted left front rectangle shows a $13 \mathrm{~cm}$ difference with the real one, whereas the high building rectangle results $13 \mathrm{~cm}$ narrower than the real one.

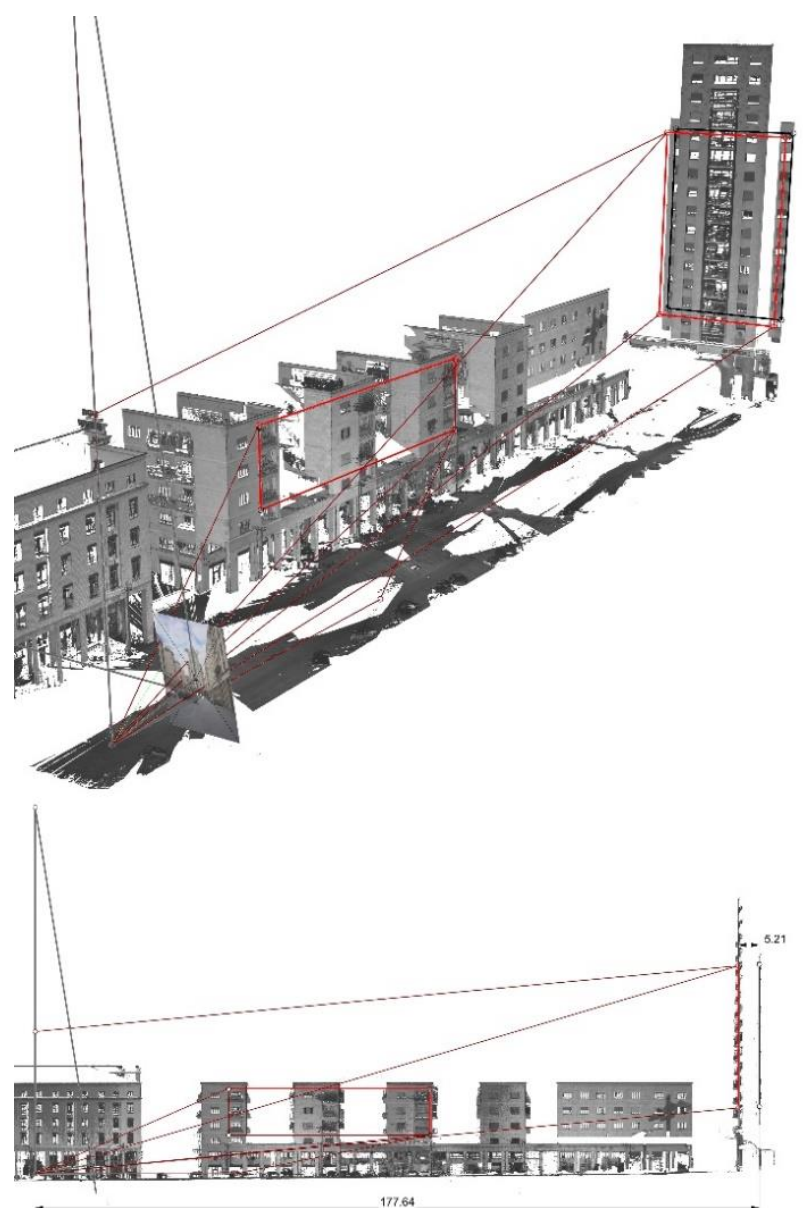

Figure 16: Perspective and front view of the outer orientation of the image taken with a standard mirrorless camera.

Major discrepancies resulted in the position of rectangles: after the superimposition of the left front rectangles, the restituted rectangle of the tall building appeared $5.20 \mathrm{~m}$ closer to the camera than the real one, with the camera at a distance of $177 \mathrm{~m}$ from this rectangle (Fig. 16).

This image has been oriented with SfM photogrammetric processes and the position of the point of view has been calculated with reference to the laser scanning data (Fig. 17).

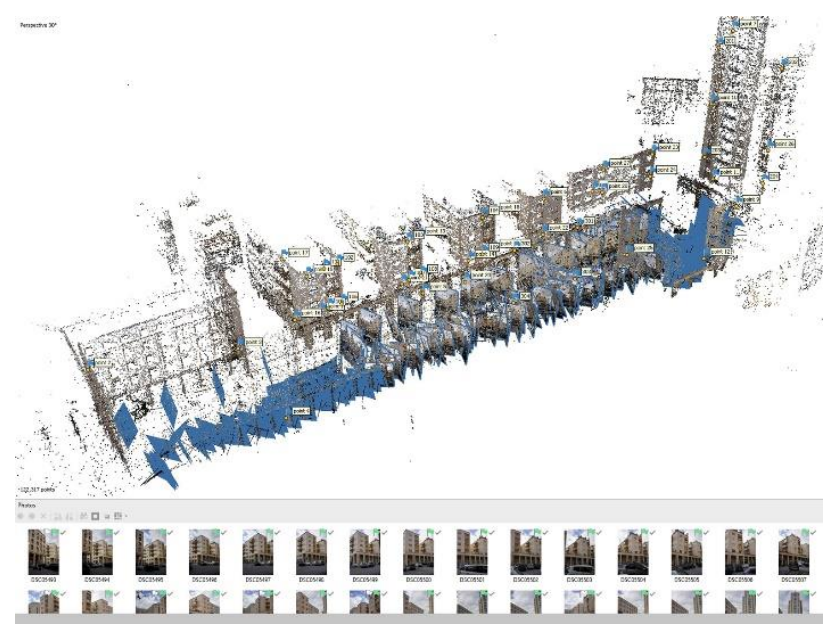

Figure 17: SfM photogrammetric model of the case study. 
The coordinates of the point of view estimated by the SfM tools and those resulting from the restitution process resulted: $\mathrm{dx}=103 \mathrm{~cm}, \mathrm{dy}=21 \mathrm{~cm}, \mathrm{dz}=4 \mathrm{~cm}$.

\section{CONCLUSIONS}

Perspective restitution with digital tools has strongly reduced the errors and inaccuracy of the restitution made with traditional drawing tools; nonetheless its accuracy results inadequate if compared to other photogrammetric techniques.
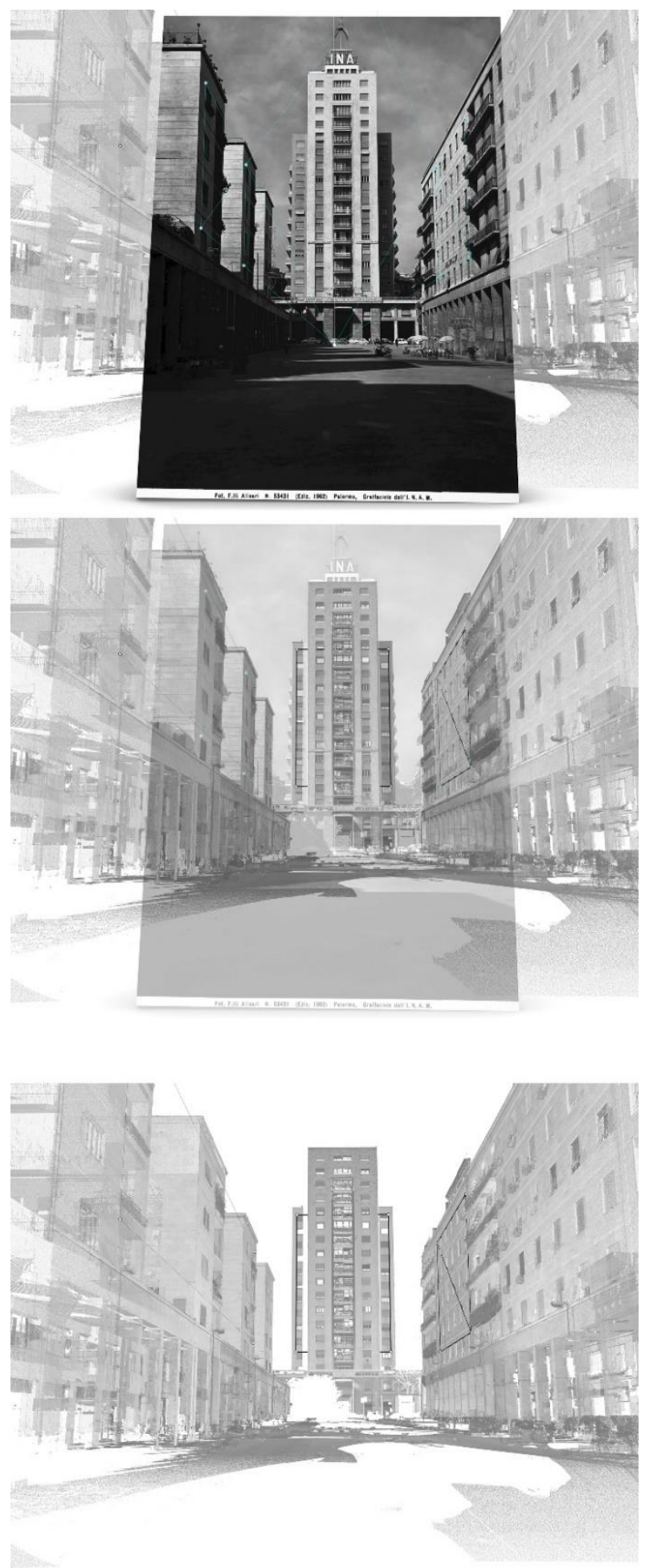

Figure 18: Visual verification of the external orientation of Alinari's image.
The paper has discussed the results of perspective restitution from images taken with a view camera, with the aim of evaluating if the restitution of a 3D scene with such images is affected by errors provoked by the movements of the rear standard.

The experiment revealed that the errors resulting from inner and outer orientation of images taken with a view camera are absolutely compatible with the errors resulting from the restitution from images taken with a standard camera.

Furthermore, the use of large format sensitive surfaces (plates, films), used by view cameras, seems to reduce the errors of restitution.

The visual verification of the inner and outer orientation of Alinari's image proves once again the accuracy of the restitution process (Fig. 18)

Further test should address the effects of the movements of the front standard, in order to evaluate its influence on the position of the principal point. The proximity of the principal point to the barycenter of the image even when the rear standard has been tilted could be probably explained by the translation of the front standard.

\section{ACKNOWLEDGEMENTS}

The author is grateful to professor Sandro Scalia, photographer and photography teacher at the Academy of Arts of Palermo for having generously offered his time, his cameras and his competence for the reported experiment. Many results of this study come from our talks on view cameras.

The image taken by Studio Alinari has been purchased by the author, that is authorized to use the image for non-profit purposes, i.e. study and research.

\section{REFERENCES}

Arslan, O., 2014. 3D Object Reconstruction from a single Image. International Journal of Environment and Geoinformatics, n. 1, pp. 21-28.

Debevec, P., Taylor, C., Malik, J., 1996. Modeling and Rendering Architecture from Photographs: a hybrid geometry and image-based approach. SIGGRAPH 1996, pp. 1-10.

Dzwierzynska, J., 2017. Establishing Base Elements of Perspective in Order to Reconstruct Architectural Buildings from Photographs. WMESS 95, pp. 1-7.

Fallavollita, F., Migliari, R., Salvatore, M., 2013. Monge e il problema del vertice di piramide: una applicazione alla restituzione di quote e volumi da una fotografia del 1892, in DisegnareCon, Vol. 6, n. 12 (2013), pp. IX/1-9.

Ramon-Constanti, A., Gomez, A., 2020. Perspective restitution from a photograph. EGA, 146, pp. 146-156.

Van den Heuvel, F.A., 1998. 3D reconstruction from a single image using geometric constraints. ISPRS Journal of Photogrammetry and Remote sensing, n. 53, pp. 354-368. 